\title{
Lattice dynamics in the thermoelectric Zintl compound $\mathrm{Yb}_{14} \mathrm{MnSb}_{11}$
}

\author{
A. Möchel, ${ }^{1,2}$ I. Sergueev,${ }^{3}$ H.-C. Wille,${ }^{4}$ F. Juranyi,${ }^{5}$ H. Schober, ${ }^{6,7}$ W. Schweika, ${ }^{1}$ S. R. Brown, ${ }^{8}$ \\ S. M. Kauzlarich, ${ }^{8}$ and R. P. Hermann ${ }^{1,2, *}$ \\ ${ }^{1}$ Jülich Centre for Neutron Science (JCNS) and Peter Grünberg Institut (PGI), JARA-FIT, Forschungszentrum Jülich GmbH, \\ D-52425 Jülich, Germany \\ ${ }^{2}$ Faculté des Sciences, Université de Liège, B-4000 Liège, Belgium \\ ${ }^{3}$ European Synchrotron Radiation Facility, F-38043 Grenoble Cedex, France \\ ${ }^{4}$ Deutsches Elektronen-Synchrotron, D-22607 Hamburg, Germany \\ ${ }^{5}$ Laboratory for Neutron Scattering, ETH Zürich and Paul Scherrer Institut, CH-5232 Villigen-PSI, Switzerland \\ ${ }^{6}$ Institut Laue-Langevin, 6 rue Jules Horowitz, F-38042 Grenoble Cedex 9, France \\ ${ }^{7}$ Université Joseph Fourier, Unité de Formation et de Recherche (UFR) de Physique, F-38041 Grenoble Cedex 9, France \\ ${ }^{8}$ Department of Chemistry, University of California, One Shields Avenue, Davis, California 95616, USA
}

(Received 8 July 2011; published 16 November 2011)

\begin{abstract}
The density of phonon states in the thermoelectric material $\mathrm{Yb}_{14} \mathrm{MnSb}_{11}$ has been studied first by inelastic neutron scattering and second in an element-specific way by nuclear inelastic $\mathrm{x}$-ray scattering. The low sound velocity of $1880(50) \mathrm{m} / \mathrm{s}$ as obtained from the density of phonon states can be identified as an important reason for the low heat transport in this system. The high melting temperature of $\mathrm{Yb}_{14} \mathrm{MnSb}_{11}$ contrasts with the low energy of all phonons $(<25 \mathrm{meV})$ and relates to an unusual lack of softening of phonon modes with temperature, when comparing the phonon density of states observed at ambient temperatures and at $1200 \mathrm{~K}$. We have also measured the density of phonon states of the related $\mathrm{Eu}_{14} \mathrm{MnSb}_{11}$ compound and of the thermoelectric Zintl phase $\mathrm{Zn}_{4} \mathrm{Sb}_{3}$ in order to compare with related thermodynamic properties of $\mathrm{Yb}_{14} \mathrm{MnSb}_{11}$ and to elucidate the different mechanisms of the heat conductivity reduction in Zintl phases.
\end{abstract}

DOI: $10.1103 /$ PhysRevB.84.184303

PACS number(s): 63.20.D-, 72.20.Pa, 76.80.+y, 61.05.C-

\section{INTRODUCTION}

The interest for environment-friendly power generation and conversion with thermoelectric materials has constantly increased during the last 20 years. Thermoelectric devices convert a heat flow into electricity and vice versa. Next to radioisotope thermoelectric generators used in spacecraft, ${ }^{1}$ a new potential application is to convert waste heat, e.g., from the exhaust gas of automobiles, into electricity. ${ }^{1}$ Due to the rather low efficiency of currently available thermoelectric devices, such waste-heat converters are still rare., ${ }^{2,3}$ The efficiency of a thermoelectric material is expressed by the dimensionless figure of merit $Z T=\left(\sigma \alpha^{2} T\right) / \kappa$, with the electrical conductivity, $\sigma$, the Seebeck coefficient, $\alpha$, and the thermal conductivity, $\kappa$. Slack summarized this requirement in stating that a good thermoelectric material should behave as both an electron crystal and a phonon glass. ${ }^{4}$ Large device efficiency requires both materials with high $Z T$ values in the requested temperature range ${ }^{5}$ and a large temperature difference. ${ }^{6}$ Because the electrical and thermal properties are temperature dependent, the efficiency of a device can be increased by combining different thermoelectric materials, which are operating in their high-efficiency temperature range only. ${ }^{6}$ For such segmented thermoelectric generators, materials with high efficiency in different temperature ranges and high compatibility factors are required. $\mathrm{Yb}_{14} \mathrm{MnSb}_{11}$ shows a high compatibility factor to existing high-temperature thermoelectric materials. ${ }^{7}$

Zintl phases are promising thermoelectric materials for thermoelectric devices ${ }^{1,7,8}$ because of the low thermal conductivity and good electrical properties found in many of such compounds. Zintl phases are intermetallic compounds in which the electronegative ion is structure forming and, due to the rich chemistry of Zintl phases, the electrical and thermodynamic properties can be easily tuned by doping. ${ }^{8}$ The low thermal conductivity is explained by many different mechanisms. ${ }^{8}$ For example, in cage structures such as skutterudites and clathrates, the thermal conductivity is reduced by introducing filler atoms which disturb the thermal transport, ${ }^{9,10}$ although details are still debated. ${ }^{11,12}$ Furthermore, in partially filled cages or in systems with partial structural disorder, such as glassy interstitials ${ }^{13}$ in $\mathrm{Zn}_{4} \mathrm{Sb}_{3}$, phonon scattering on crystal defects can reduce the thermal conductivity. ${ }^{14}$ Vibrational subunits that exhibit localized phonon modes, ${ }^{15}$ which are crossing the acoustic modes, can also yield a low thermal conductivity, e.g., again in $\mathrm{Zn}_{4} \mathrm{Sb}_{3}$. More relevant herein, Zintl phases with a large unit cell volume and a complex crystal structure typically exhibit low thermal conductivity. ${ }^{8}$

The Zintl phase $\mathrm{Yb}_{14} \mathrm{MnSb}_{11}$ provides good electronic properties in the high-temperature range ( $>900 \mathrm{~K}$ ), properties similar to those of a heavily doped semiconductor, and it exhibits a low thermal conductivity of $\sim 0.8 \mathrm{~W} /(\mathrm{m} \mathrm{K})$ at $300 \mathrm{~K}$. The thermal conductivity is nearly temperature independent ${ }^{7}$ between 300 and $1200 \mathrm{~K}$, being on the same order of magnitude as that of a glass ${ }^{7}$ and low compared to other thermoelectric materials such as tellurides, e.g., $\mathrm{Sb}_{2} \mathrm{Te}_{3}$ or $\mathrm{Bi}_{2} \mathrm{Te}_{3}$, and skutterudites, e.g., $\mathrm{YbFe}_{4} \mathrm{Sb}_{12}$, which exhibit $^{13,16}$ thermal conductivities of $\sim 2 \mathrm{~W} /(\mathrm{m} \mathrm{K})$ at $300 \mathrm{~K}$. The melting temperature of $\mathrm{Yb}_{14} \mathrm{MnSb}_{11}$ cannot be found in the literature but the material ${ }^{7}$ is solid up to $1275 \mathrm{~K}$. Because of the high melting temperature, and the good electrical properties and the low thermal conductivity up to $1200 \mathrm{~K}$, it is a promising thermoelectric material for high-temperature applications, e.g., in radioisotope thermoelectric generators. 
The figure of merit of $\mathrm{Yb}_{14} \mathrm{MnSb}_{11}$ exceeds ${ }^{7}$ that of the currently used $p$-doped $\mathrm{SiGe}$ by more than $60 \%$. It was suggested that the low thermal conductivity is related to the complex unit cell and the large mass differences of the atoms in the structure. ${ }^{8} \mathrm{Yb}_{14} \mathrm{MnSb}_{11}$ exhibits a tetragonal unit cell with $I 4_{1} /$ acd space group and the unit cell contains 208 atoms, ${ }^{17}$ for a unit cell volume of $\sim 6060 \AA^{3}$, and the volume of the primitive unit cell is $\sim 3030 \AA^{3}$. According to the formalism derived by Toberer et al., ${ }^{8}$ the heat capacity, and therefore the thermal conductivity, is inversely proportional to the volume of the primitive unit cell as the relative heat capacity is equally divided among all phonon modes. The small ratio of the number of acoustic to optical modes results in a small contribution to the thermal transport, because the optical phonon branches are typically flat and therefore the heat transport through the optical phonons is low. With 104 atoms per primitive unit cell, $\mathrm{Yb}_{14} \mathrm{MnSb}_{11}$ exhibits 3 acoustic and 309 optical phonon modes. Because the heat capacity of the acoustic modes is decreased compared to systems with fewer optical modes, the contribution of the heat capacity to the thermal transport is reduced and therefore the thermal conductivity is lowered. The large mass contrast between the atoms in the structure also reduces the role of the optical modes in the thermal transport. ${ }^{8}$ The mass ratio of $\mathrm{Yb}$ and $\mathrm{Sb}$ is 1.42 , which is close to the typical criterion of 1.5 , above which the heat transport through the optical modes can be neglected. ${ }^{18}$

The Debye temperature in $\mathrm{Yb}_{14} \mathrm{MnSb}_{11}$ of $160(10) \mathrm{K}$, was extracted from specific heat capacity data ${ }^{19}$ and it is surprising that the melting temperature is at least eight times higher than the Debye temperature. The low Debye temperature correlates with a low sound velocity and this is a second reason for the low thermal conductivity in $\mathrm{Yb}_{14} \mathrm{MnSb}_{11}$, next to the reduction of the effective heat capacity contribution to the thermal conductivity ascribed ${ }^{8}$ to the large unit cell volume. Because of the low Debye temperature it is expected that the phonon modes are in a low-energy range with a small phonon mode cutoff energy, as systems with a low Debye temperature typically exhibit phonon modes in a low-energy range. There are, however, notable exceptions, such as vitreous silica, which exhibits $^{20}$ a Debye temperature of $487 \mathrm{~K}$ and phonon modes up to $170 \mathrm{meV}$. Thus it is important to directly characterize the lattice vibrations and not to rely simply on a Debye model. Furthermore, an optical phonon softening below the Curie temperature of $53 \mathrm{~K}$ was observed, which results from a Kondo-phonon coupling. ${ }^{21}$ This behavior was not investigated herein, because the energy resolution of the used methods was much larger than the expected softening. So far there are no lattice dynamics calculations of $\mathrm{Yb}_{14} \mathrm{MnSb}_{11}$, which is likely due to the large and complex unit cell.

Herein the lattice dynamics, in particular the density of phonon states, is investigated experimentally. Temperaturedependent $\mathrm{x}$-ray diffraction, in particular the thermal expansion, was investigated in $\mathrm{Yb}_{14} \mathrm{MnSb}_{11}$. The total density of phonon states (DPS) was determined by inelastic neutron scattering (INS). In order to obtain the element-specific partial density of phonon states, nuclear inelastic scattering (NIS) measurements were carried out. For comparison the DPS in the isostructural compound $\mathrm{Eu}_{14} \mathrm{MnSb}_{11}$ was also measured by NIS. It is not practical to measure the density of phonon states in $\mathrm{Eu}_{14} \mathrm{MnSb}_{11}$ by inelastic neutron scattering, due to the large neutron absorption cross section of Eu. The ${ }^{121} \mathrm{Sb}$ and ${ }^{151} \mathrm{Eu}$ nuclear resonances can, however, be used to probe the density of phonon states by NIS. Because the Yb DPS cannot currently be measured by NIS, the ${ }^{151}$ Eu density of phonon states was used as a representative value for the Yb DPS in $\mathrm{Yb}_{14} \mathrm{MnSb}_{11}$. By combining the INS and NIS measurements, a complete insight in the partial density of phonon states of $\mathrm{Yb}_{14} \mathrm{MnSb}_{11}$ is obtained. For further comparisons, we also investigate $\mathrm{Zn}_{4} \mathrm{Sb}_{3}$, another interesting thermoelectric material with a large primitive unit cell volume ${ }^{22}$ of $\sim 540 \AA^{3}$, which is, however, five times smaller than $\mathrm{Yb}_{14} \mathrm{MnSb}_{11} . \mathrm{Zn}_{4} \mathrm{Sb}_{3}$ has a thermal conductivity ${ }^{1,22,23}$ between 0.7 and $1.3 \mathrm{~W} /(\mathrm{m} \mathrm{K})$ at $300 \mathrm{~K}$, a value similar to that in $\mathrm{Yb}_{14} \mathrm{MnSb}_{11}$. The ZintlKlemm concept can be applied to the idealized $\mathrm{Zn}_{13} \mathrm{Sb}_{10}$ structure ${ }^{24}$ and therefore $\mathrm{Zn}_{4} \mathrm{Sb}_{3}$ is regarded as a Zintl phase herein. However, the electronic structure does not show the ionic description expected from the Zintl-Klemm concept, but delocalized multicenter bonding. ${ }^{1}$ The origin of the low thermal conductivities in $\mathrm{Yb}_{14} \mathrm{MnSb}_{11}$ and $\mathrm{Zn}_{4} \mathrm{Sb}_{3}$ is likely different: In $\mathrm{Zn}_{4} \mathrm{Sb}_{3}$ it was assumed that the main reason for the low thermal conductivity is a small phonon mean free path due to vibrational subunits ${ }^{15}$ or disordered $\mathrm{Zn}$ interstitials. ${ }^{13}$ We expect that in $\mathrm{Yb}_{14} \mathrm{MnSb}_{11}$ the low thermal conductivity is due to a low sound velocity and low effective heat capacity contributions to the thermal conductivity. Our data provide a comprehensive basis to discuss the different mechanisms of the low thermal conductivity in Zintl phases.

\section{EXPERIMENT}

The synthesis of polycrystalline $\mathrm{Yb}_{14} \mathrm{MnSb}_{11}$, used in all measurements reported herein, is described in Ref. 17. Temperature-dependent diffraction measurements were carried out between 15 and $300 \mathrm{~K}$ on a Huber powder diffractometer with $\mathrm{Cu} K_{\alpha 1}$ radiation using an image plate Guinier camera and with synchrotron radiation at 13 and $300 \mathrm{~K}$ at the 6-ID-D high-energy station at the Advanced Photon Source (APS) by using a wavelength of $0.124269 \AA$ and an imaging plate detector. Le Bail fits ${ }^{25}$ using the profile-matching method of FULLPROF ${ }^{26}$ were carried out in order to obtain the lattice parameters from the diffraction patterns. Scattering vector, $Q$, and energy-dependent inelastic neutron scattering measurements were performed at the cold-neutron time-offlight spectrometer FOCUS at the Paul Scherrer Institut (PSI) at room temperature. The measurement took place at the phonon annihilation side with an incident wavelength of $2.3 \AA$ in an energy range between 0 and $40 \mathrm{meV}$ and in the $Q$ range 2-6 $\AA^{-1}$ on average. The energy resolution varied between 1 and $5 \mathrm{meV}$ depending on the energy transfer; e.g., at an energy transfer of 5 and $25 \mathrm{meV}$ the resolutions were 1.5 and $4.3 \mathrm{meV}$, respectively. The background from an Al hollowcylinder sample holder was subtracted and the data were normalized to vanadium scattering. The data reduction was carried out using DAVE. ${ }^{27}$ INS measurements were performed at the IN6 spectrometer at the Institut Laue-Langevin (ILL) at 300,750 , and $1200 \mathrm{~K}$ with an incident wavelength of $5.12 \AA$. Due to the strong inelastic scattering of the $\mathrm{Nb}$ sample holder the data are reliable below $15 \mathrm{meV}$ only. The 
data were reduced using LAMP. $^{28}$ NIS was measured with the ${ }^{121} \mathrm{Sb}$ resonance on $\mathrm{Yb}_{14} \mathrm{MnSb}_{11}$, and on $\mathrm{Eu}_{14} \mathrm{MnSb}_{11}$ and $\mathrm{Zn}_{4} \mathrm{Sb}_{3}$ for comparison, at the European Synchrotron Radiation Facility (ESRF) ID22N station in 16-bunch mode, with the samples at $25 \mathrm{~K}$. Measurements with the ${ }^{151} \mathrm{Eu}$ resonance on the isostructural compound $\mathrm{Eu}_{14} \mathrm{MnSb}_{11}$ were performed at $58 \mathrm{~K}$ at the ID18 station, ESRF. The same $\mathrm{Zn}_{4} \mathrm{Sb}_{3}$ and $\mathrm{Eu}_{14} \mathrm{MnSb}_{11}$ samples as in Refs. 15 and 29, respectively, were used.

\section{RESULTS AND DISCUSSION}

\section{A. Structure}

The crystal structure of $\mathrm{Yb}_{14} \mathrm{MnSb}_{11}$ is shown in Fig. 1. The structure consists of 8 units comprising $14 \mathrm{Yb}^{2+}$ cations, $1 \mathrm{MnSb}_{4}{ }^{9-}$ tetrahedron (indicated in Fig. 1 in yellow), $1 \mathrm{Sb}_{3}{ }^{7-}$ polyatomic anion, and $4 \mathrm{Sb}^{3-}$ anions. ${ }^{17}$ The diffraction pattern at $15 \mathrm{~K}$ in Fig. 2 reveals no secondary phases. The temperature dependence of the lattice parameters, see Fig. 3, were obtained by a profile matching of the diffraction pattern. Due to the low-symmetry space group and the complex diffraction pattern, a refinement of the positions of the atoms in the unit cell, of the atomic site occupation, and of the thermal mean square displacements was not possible for the powder samples. Recently, the mean square displacements were refined from $\mathrm{X}$-ray diffraction patterns and published in Ref. 30 .

The values of the lattice parameters at $300 \mathrm{~K}$ of 16.6140 (2) and 21.9925(3) $\AA$ for $a$ and $c$, respectively, are in good agreement with the 13 and $300 \mathrm{~K}$ values obtained by synchrotron radiation diffraction (see black points in Fig. 3), and the values are also in good agreement with published values. ${ }^{30}$ The Bragg $R_{B}$ and the $R_{f}$ factor of the profile matching were 0.3 and $0.4 \%$, respectively, for all temperatures. A density, $\rho$, of $8.35(2) \mathrm{g} / \mathrm{cm}^{3}$ was obtained from the lattice parameter at $300 \mathrm{~K}$. The thermal expansion coefficient, $\alpha$, was obtained from a model of the temperature dependence of the lattice parameter $a$, with a third-order polynomial function, $a_{m}(T)$, in order to reduce noise in the data and by using $\alpha_{a}=\left[d a_{m}(T) / d T\right] / a_{m}(300 \mathrm{~K})$; see Fig. 3. The difference between the modeled lattice parameter and the data was

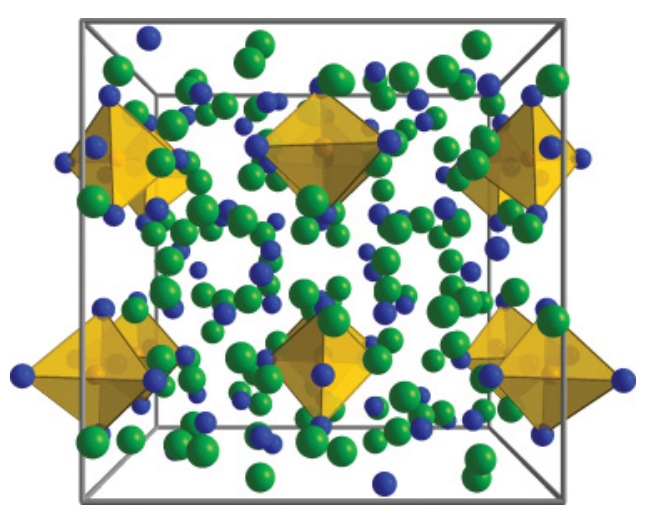

FIG. 1. (Color online) Crystal structure of $\mathrm{Yb}_{14} \mathrm{MnSb}_{11}$ and $\mathrm{Eu}_{14} \mathrm{MnSb}_{11}$. Large (green), medium (blue), and small (yellow) spheres represent $\mathrm{Yb}$ or $\mathrm{Eu}, \mathrm{Sb}$, and $\mathrm{Mn}$ atoms, respectively.

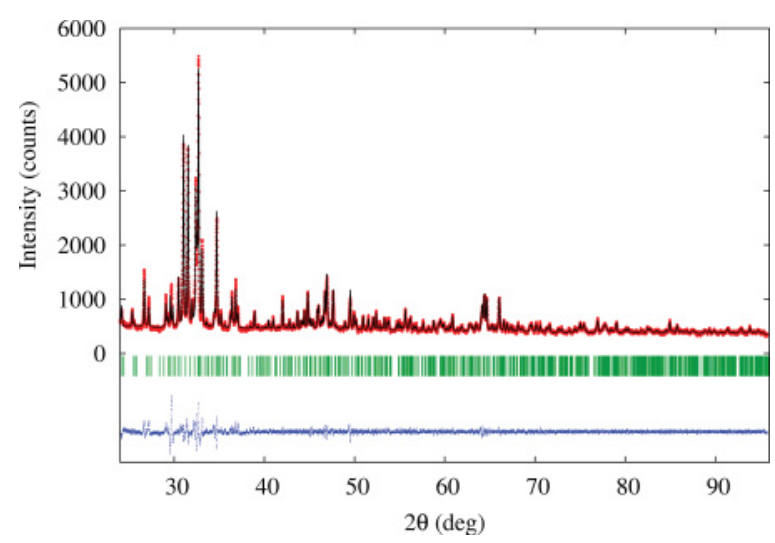

FIG. 2. (Color online) Characterization of the $\mathrm{Yb}_{14} \mathrm{MnSb}_{11}$ powder sample by xray diffraction at $15 \mathrm{~K}$. The red and black lines (top) indicate the measured pattern and the Le Bail fit, respectively, the green tics (middle) indicates the peak positions, and the blue line (bottom) the difference between measurement and the Le Bail fit.

less than $8 \times 10^{-4} \AA$. The temperature dependence at low temperatures of the thermal expansion coefficient from the $c$ lattice parameter cannot be calculated due to insufficient precision in the lattice parameter values at low temperatures. Only the 100-300-K region was fitted linearly (see Fig. 3), and a thermal expansion of $19.4(2) \times 10^{-6} \mathrm{~K}^{-1}$ was obtained by $\alpha_{c}=[d c(T) / d T] / c(300 \mathrm{~K})$. The mean value of the thermal

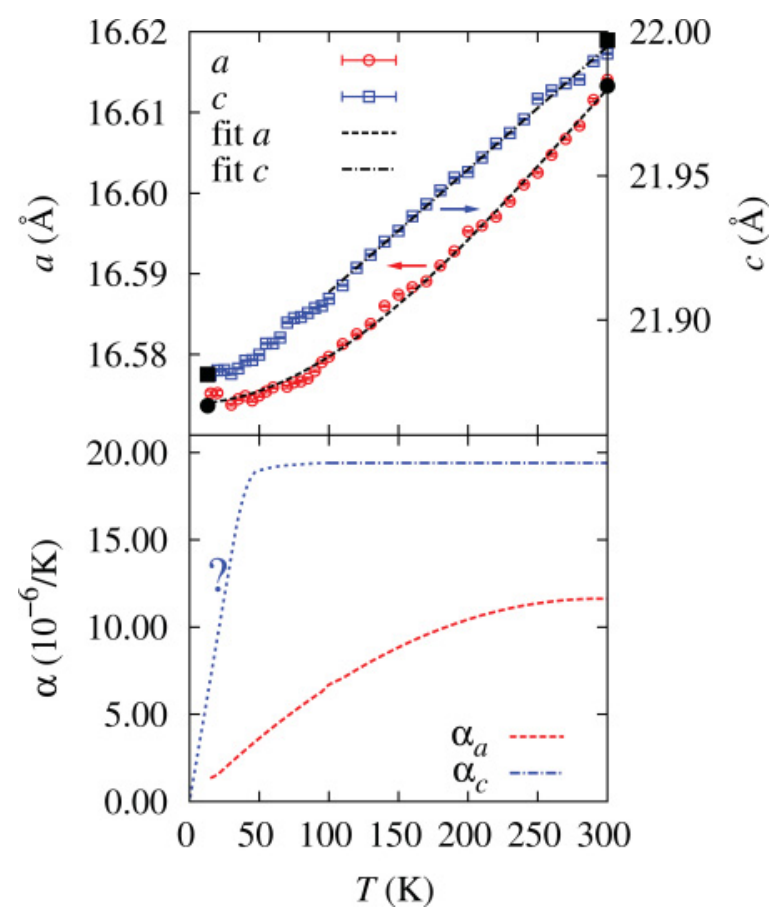

FIG. 3. (Color online) (top) Temperature dependence of the lattice parameters $a$ (red) and $c$ (blue) obtained from x-ray powder diffraction and the fit of the modulated $a_{m}$ for $a$ and the linear fit for $c$. The error bars are in the order of the point size. The black points indicate the values obtained from synchrotron radiation diffraction at 13 and $300 \mathrm{~K}$. (bottom) Anisotropy of the thermal expansion, $\alpha$, in $\mathrm{Yb}_{14} \mathrm{MnSb}_{11}$ along the $a$ and $c$ direction obtained by $a_{m}(T)$ for $a$ and the linear fit for $c$. The dotted line indicates a possible low temperature dependence of the thermal expansion coefficient for $c$. 
expansion coefficient at $300 \mathrm{~K}$, obtained by $\alpha=\left(2 \alpha_{a}+\alpha_{c}\right) / 3$, is $14.2(4) \times 10^{-6} \mathrm{~K}^{-1}$. This is in fair agreement with the value of $16.6 \times 10^{-6} \mathrm{~K}^{-1}$ obtained $^{31}$ by dilatometry measurements in the high-temperature region between 323 and $1273 \mathrm{~K}$. The Grüneisen parameter $\gamma=3 \alpha B V_{m} / C_{V}$ is determined by the bulk modulus, $B$, the molar volume, $V_{m}$, and the specific heat capacity, $C_{V}$. The bulk modulus, $B=38(5) \mathrm{GPa}$, was extracted from the sound velocity of $1880(50) \mathrm{m} / \mathrm{s}$ obtained from NIS (see below) by taking the Poisson ratio ${ }^{32}$ of 0.24 into account. With the specific heat capacity of $650 \mathrm{~J} \mathrm{~K}^{-1} \mathrm{~mol}^{-1}$, which is obtained by the Dulong-Petit law and is in good agreement with the value obtained by NIS (see discussion below), a $\gamma$ value of 1.2(1) was obtained at $300 \mathrm{~K}$. For $\mathrm{Zn}_{4} \mathrm{Sb}_{3}$ a Grüneisen parameter of $1.35(5)$ at $300 \mathrm{~K}$ was calculated from literature values. ${ }^{15,23,33}$ The Grüneisen parameter indicates anharmonicity in the lattice dynamics and the typical values for semiconductors are between 1 and $2 .{ }^{34}$ The thermal conductivity is connected to the anharmonicity in the material and therefore the Grüneisen parameter is a useful parameter for the comparison of thermoelectric materials. In the thermoelectric material $\mathrm{AgSbTe}_{2}$ an extremely high value of $\gamma=2.05$ was found, indicating a large anharmonicity, which can explain the low thermal conductivity of $0.7 \mathrm{~W} /(\mathrm{m} \mathrm{K})$ found in this material. ${ }^{35}$ The values found here for $\mathrm{Yb}_{14} \mathrm{MnSb}_{11}$ and $\mathrm{Zn}_{4} \mathrm{Sb}_{3}$ are in a $\gamma$ range between 1.2 and 1.5 , which is typical for thermoelectric materials like skutterudites ${ }^{36}$ and lead tellurides. ${ }^{35}$

\section{B. Density of phonon states}

In order to study the density of phonon states in $\mathrm{Yb}_{14} \mathrm{MnSb}_{11}$, INS measurements were carried out. The $Q$

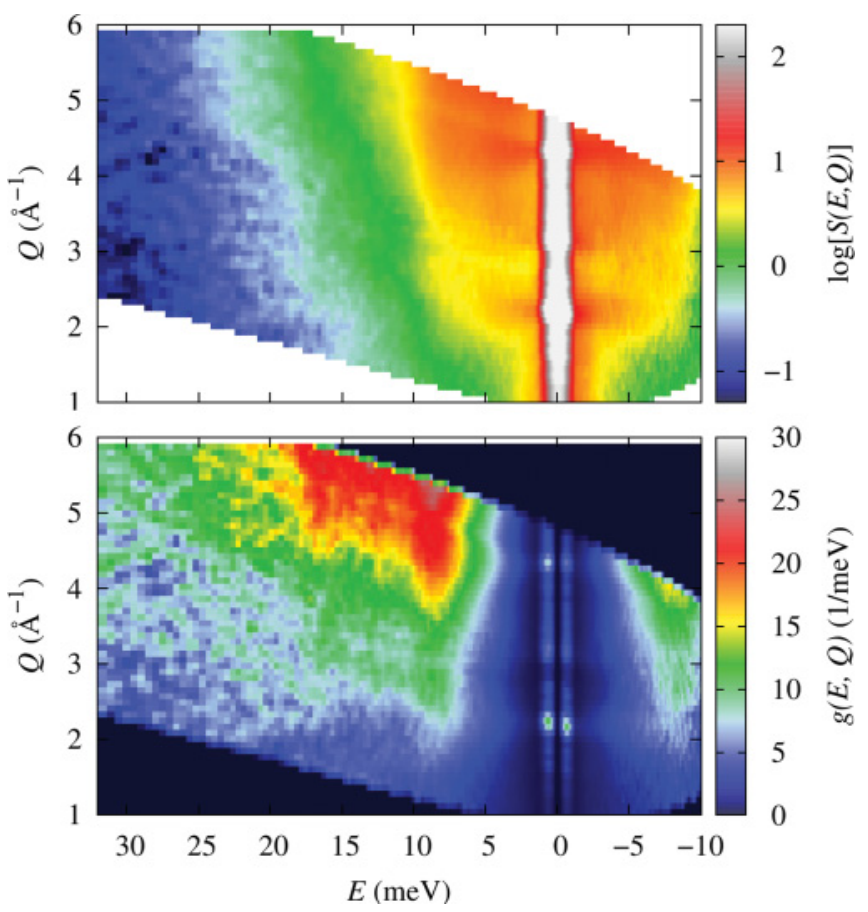

FIG. 4. (Color online) (top) The $Q$ and energy dependence of the inelastic neutron scattering on a $\mathrm{Yb}_{14} \mathrm{MnSb}_{11}$ powder sample at $300 \mathrm{~K}$. (bottom) The $Q$ dependence of the extracted density of phonon states at $300 \mathrm{~K}$. and energy dependence of the dynamical structure factor, $S(E, Q)$, of $\mathrm{Yb}_{14} \mathrm{MnSb}_{11}$ measured at FOCUS is shown in the top panel of Fig. 4. The normal $Q^{2}$ dependence of phonon scattering for incoherent scatterers was observed. ${ }^{37}$ The intensity decreases with increasing energy, and the powder average of the $Q$-dependent density of phonon states, $g(E, Q)$, was obtained after subtraction of the elastic line and correction by the Bose-Einstein factor ${ }^{37}$ (see bottom panel of Fig. 4), with

$$
g(E, Q)=\frac{S(E, Q) E}{\exp \left[E /\left(k_{B} T\right)\right]-1} .
$$

A relatively sharp phonon band is observed at $\sim 10 \mathrm{meV}$ and a broad band is observed between 12 and $20 \mathrm{meV}$. Modes are observed only in the energy region below $25 \mathrm{meV}$ (see also NIS data below), as expected qualitatively from the low Debye temperature $^{19}$ of $160(10) \mathrm{K}$ as a typical behavior. The total DPS from this measurement obtained by integrating $g(E, Q)$ over $Q$ in the incoherent approximation is discussed below in comparison with the partial DPS obtained from NIS.

The temperature dependence of $S(E, Q)$ was obtained at 300,750 , and $1200 \mathrm{~K}$ on $\mathrm{Yb}_{14} \mathrm{MnSb}_{11}$ at the IN6 spectrometer (see Fig. 5). In order to verify that no structural change occurred upon heating, neutron diffraction patterns were obtained from integration over the elastic line (see Fig. 6) in comparison with the diffraction pattern obtained from the FOCUS measurement and with a simulated neutron diffraction pattern calculated with FULLPROF using the lattice parameter at $300 \mathrm{~K}$ obtained from $\mathrm{x}$-ray diffraction. No foreign peaks from a second phase have been observed at any temperature. The shift of the peaks to lower values with increasing temperature is due to the thermal expansion. The reflections were indexed and the positions were fitted using a Gaussian profile. The

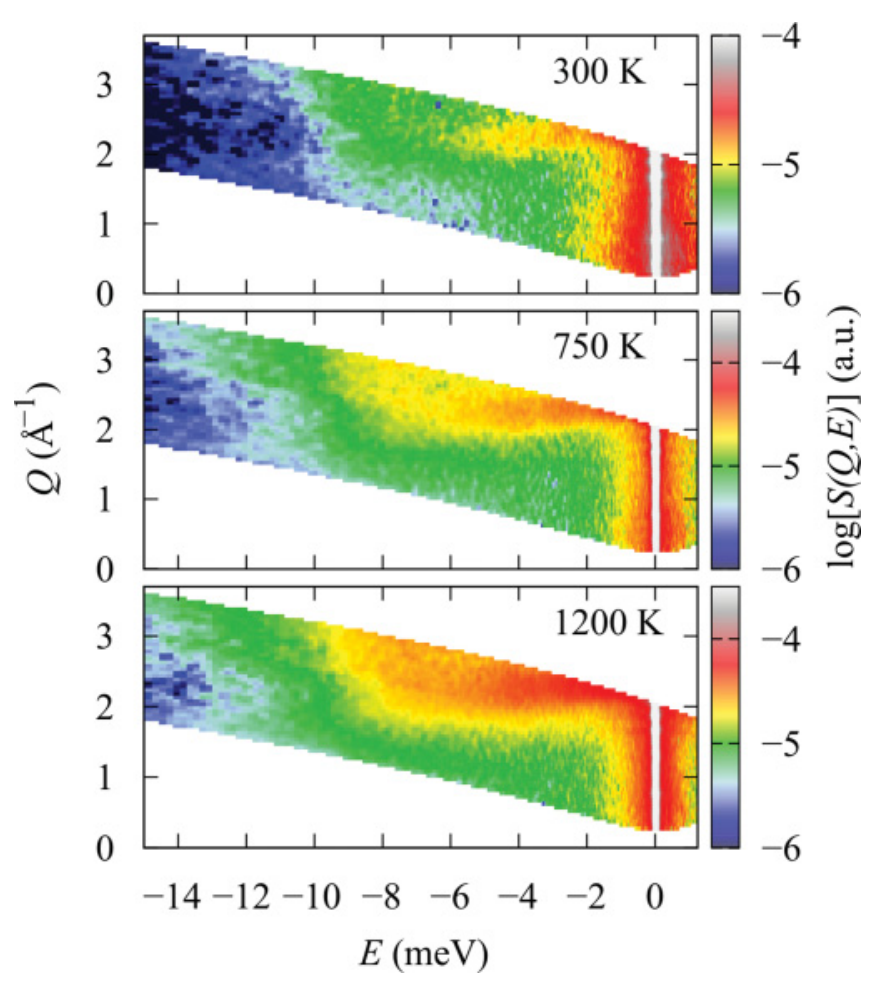

FIG. 5. (Color online) $S(E, Q)$ of a $\mathrm{Yb}_{14} \mathrm{MnSb}_{11}$ powder sample measured at the IN6 spectrometer at 300, 750, and $1200 \mathrm{~K}$. 


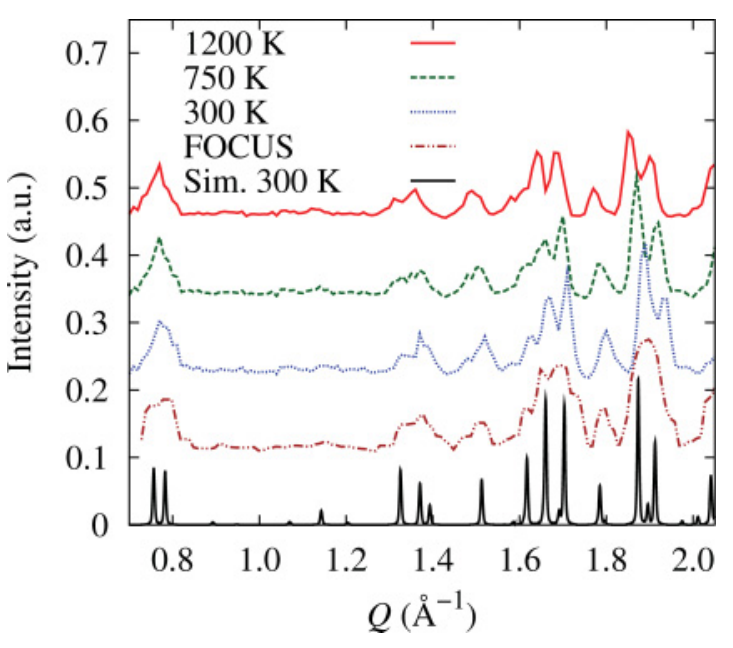

FIG. 6. (Color online) The neutron diffraction pattern of $\mathrm{Yb}_{14} \mathrm{MnSb}_{11}$ obtained from the measurement at IN6 at 300, 750, and $1200 \mathrm{~K}$ and at FOCUS at $300 \mathrm{~K}$, compared to the simulated neutron diffraction pattern (Sim.) calculated with FULLPROF with the lattice parameter at $300 \mathrm{~K}$ obtained by $\mathrm{x}$-ray diffraction.

$a / c$ lattice parameters were obtained as $16.56(5) / 21.99(5)$, $16.67(5) / 22.15(5)$, and $16.78(5) / 22.33(5) \AA$ for 300,750 , and $1200 \mathrm{~K}$, respectively. The $300-\mathrm{K}$ values are in good agreement with the values obtained by x-ray diffraction of 16.6140(2) and 21.9925(3) $\AA$ for $a$ and $c$, respectively. The values for 750 and $1200 \mathrm{~K}$ are in good agreement with the values calculated with the thermal expansion coefficient from x-ray diffraction for the $a / c$ lattice parameters of $16.70 / 22.19$ and $16.79 / 22.38 \AA$ A for 750 and $1200 \mathrm{~K}$, respectively. It was verified by dilatometry measurements ${ }^{31}$ that the thermal expansion coefficient is constant between 300 and $1200 \mathrm{~K}$.

Considering the IN6 data, the $Q$-dependent density of phonon states was obtained in the same way as for the FOCUS data. Due to the large contribution of the phonon states of the $\mathrm{Nb}$ sample holder at $17 \mathrm{meV}$ and the imprecision of the background subtraction, the data are sufficiently reliable only below $15 \mathrm{meV}$. In this transfer energy range, the $300-\mathrm{K}$ data are in good agreement with the FOCUS data. By integrating $g(E, Q)$ over $Q$, the neutron cross-section weighted density of phonon states, $g(E)$, was obtained (see Fig. 7). Due to thermal expansion the unit cell volume in real space increases with temperature and therefore the reciprocal lattice volume decreases. Because the probed reciprocal space volume is constant, the number of primitive Brillouin zones over which the integration of $g(E, Q)$ is carried out increases with temperature, and a corresponding correction of 2 and $4 \%$ for the 750- and $1200-\mathrm{K}$ data, respectively, was applied. A broadening of the phonon modes with temperature can be seen in the DPS and in the reduced DPS, $g(E) / E^{2}$. A decrease of the Debye level, $\lim _{E \rightarrow 0}\left[g(E) / E^{2}\right]$, is observed in the reduced DPS (see Fig. 7), which would indicate an increase in the sound velocity, $v_{s}$, with temperature, because $v_{s}^{3} \sim 1 /\left\{\lim _{E \rightarrow 0}\left[g(E) / E^{2}\right]\right\}{ }^{38}$ Such a decrease cannot be clarified by the data, due to possible imprecision in the background subtraction, the multiphonon correction, and the normalization of the DPS, but the data at hand suggest that the sound velocity does change only slightly with temperature

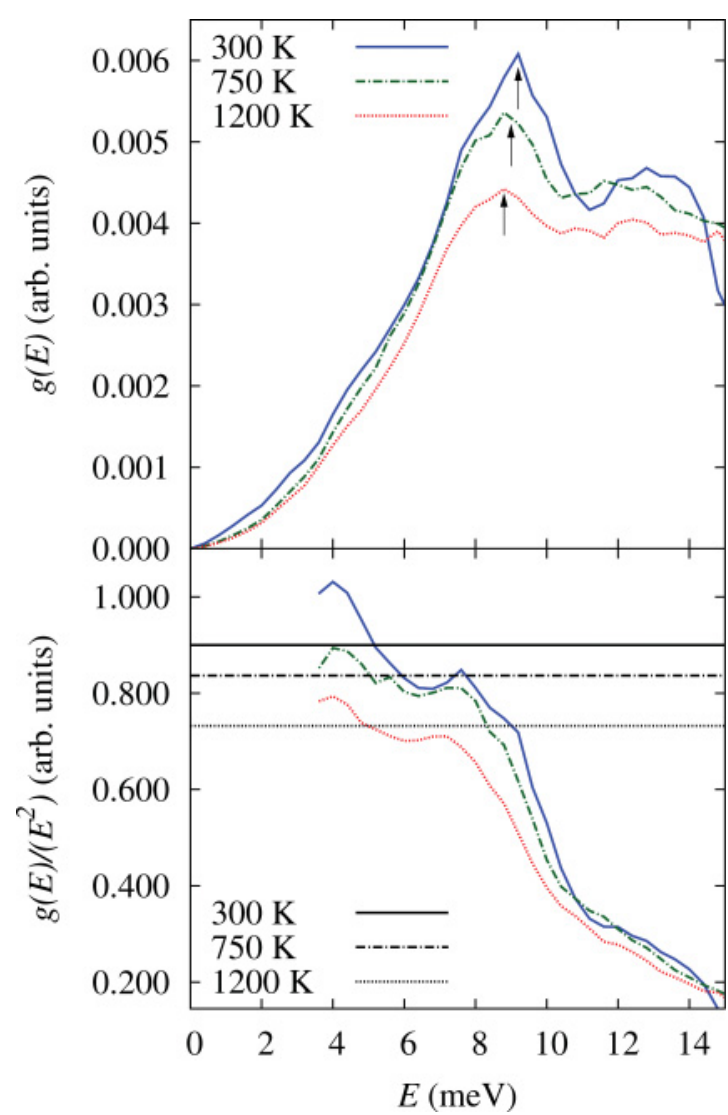

FIG. 7. (Color online) (top) Temperature dependence of the $\mathrm{Yb}_{14} \mathrm{MnSb}_{11}$ DPS measured by inelastic neutron scattering at the IN6 spectrometer. The arrows indicate the mode shift with temperature calculated from the peak position at $300 \mathrm{~K}$ with the Grüneisen parameter obtained from macroscopic measurements. (bottom) The reduced DPS. The black lines represent the Debye level at 300, 750, and $1200 \mathrm{~K}$, respectively, in the same line style.

and this behavior could correlate with the high melting temperature. In view of the unusual temperature dependence of the sound velocity, further in situ measurements, e.g., resonant ultrasound spectroscopy, at elevated temperatures are of interest. In order to measure the elastic constants by resonant ultrasound spectroscopy, a bulk sample is needed. Therefore, we tried to sinter the powder sample, but the sample changed its phase during the sintering process. From the Grüneisen parameter of $\gamma=1.2(1)$ the downshift of the phonon modes can be estimated by $\gamma=-\partial \ln E /(\partial \ln V)$, where $V$ is the unit cell volume. ${ }^{39}$ The downshift of the phonon modes at around $9 \mathrm{meV}$ with temperature is in good agreement with the macroscopic Grüneisen parameter (see Fig. 7).

With NIS, the DPS can be measured directly and isotopespecifically. The technique is described in detail elsewhere ${ }^{40}$ and results for ${ }^{121} \mathrm{Sb}$ are presented, e.g., in Ref. 41. The inelastic scattering spectra measured by NIS with the ${ }^{151} \mathrm{Eu}$ resonance in $\mathrm{Eu}_{14} \mathrm{MnSb}_{11}$ and with the ${ }^{121} \mathrm{Sb}$ resonance in $\mathrm{Eu}_{14} \mathrm{MnSb}_{11}$, in $\mathrm{Yb}_{14} \mathrm{MnSb}_{11}$, and for comparison in $\mathrm{Zn}_{4} \mathrm{Sb}_{3}$ are shown in Fig. 8, together with the respective instrumental resolution function obtained by nuclear forward scattering. The instrumental resolution was $1.3 \mathrm{meV}$ for 


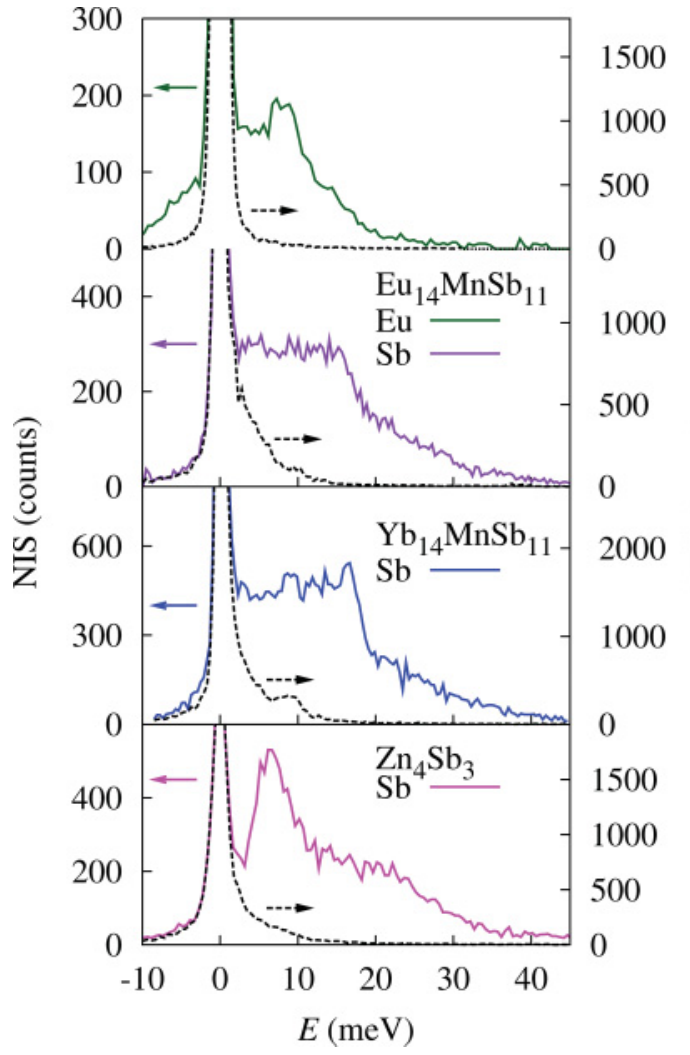

FIG. 8. (Color online) The element-specific NIS spectra measured in (top) $\mathrm{Eu}_{14} \mathrm{MnSb}_{11}$, (middle) $\mathrm{Yb}_{14} \mathrm{MnSb}_{11}$, and (bottom) $\mathrm{Zn}_{4} \mathrm{Sb}_{3}$. The black line indicates the nuclear forward scattering (NFS), which was used as the instrumental resolution function.

all measurements. ${ }^{42}$ In order to minimize the multiphonon contributions which are significant for the nuclear resonances of ${ }^{121} \mathrm{Sb}$ and ${ }^{151} \mathrm{Eu}$, the measurements were carried out at low temperatures, i.e., at 25 and $55 \mathrm{~K}$ for $\mathrm{Sb}$ and $\mathrm{Eu}$, respectively. The Eu DPS in the isostructural compound $\mathrm{Eu}_{14} \mathrm{MnSb}_{11}$ was measured because the Yb DPS could not be measured by this technique. The partial DPS was extracted after subtraction of the elastic line by the conventional procedure ${ }^{43,44}$ with a slight modification: in order to treat correctly the asymmetry of the resolution function of the ${ }^{121} \mathrm{Sb}$ NIS and to smooth the statistical noise, the data were convoluted with a symmetrical Gaussian function with a width of $1.7 \mathrm{meV}$ FWHM instead of the asymmetric instrumental function. After multiphonon correction, the partial DPS, $g(E)^{\mathrm{Sb} \text {,Eu}}$, were obtained (see Fig. 9). The Sb DPS is similar in $\mathrm{Yb}_{14} \mathrm{MnSb}_{11}$ and in $\mathrm{Eu}_{14} \mathrm{MnSb}_{11}$, indicating a similar density of phonon states, and the Eu measurement can therefore be used as representative in first approximation for the $\mathrm{Yb}$ behavior. The $\mathrm{Sb}$ phonon modes show a typical Debye behavior up to $\sim 5 \mathrm{meV}$, then increase linearly until a cutoff energy of $\sim 20 \mathrm{meV}$. The small number of observed states above $\sim 20 \mathrm{meV}$, especially the peak at $22 \mathrm{meV}$ in the ${ }^{121} \mathrm{Sb}$ data of $\mathrm{Yb}_{14} \mathrm{MnSb}_{11}$ and $\mathrm{Eu}_{14} \mathrm{MnSb}_{11}$, is due to an $\mathrm{Sb}_{2} \mathrm{O}_{3}$ impurity in these samples. From the comparison of the ${ }^{121} \mathrm{Sb}$ DPS in $\mathrm{Sb}_{2} \mathrm{O}_{3}$ (not shown) with the ${ }^{121} \mathrm{Sb}$ DPS in $\mathrm{Yb}_{14} \mathrm{MnSb}_{11}$ and $\mathrm{Eu}_{14} \mathrm{MnSb}_{11}$, impurity phases of $<10 \%$ and $<25 \%$, respectively, were obtained in good agreement with the results in Ref. 29, where samples synthesized by the same

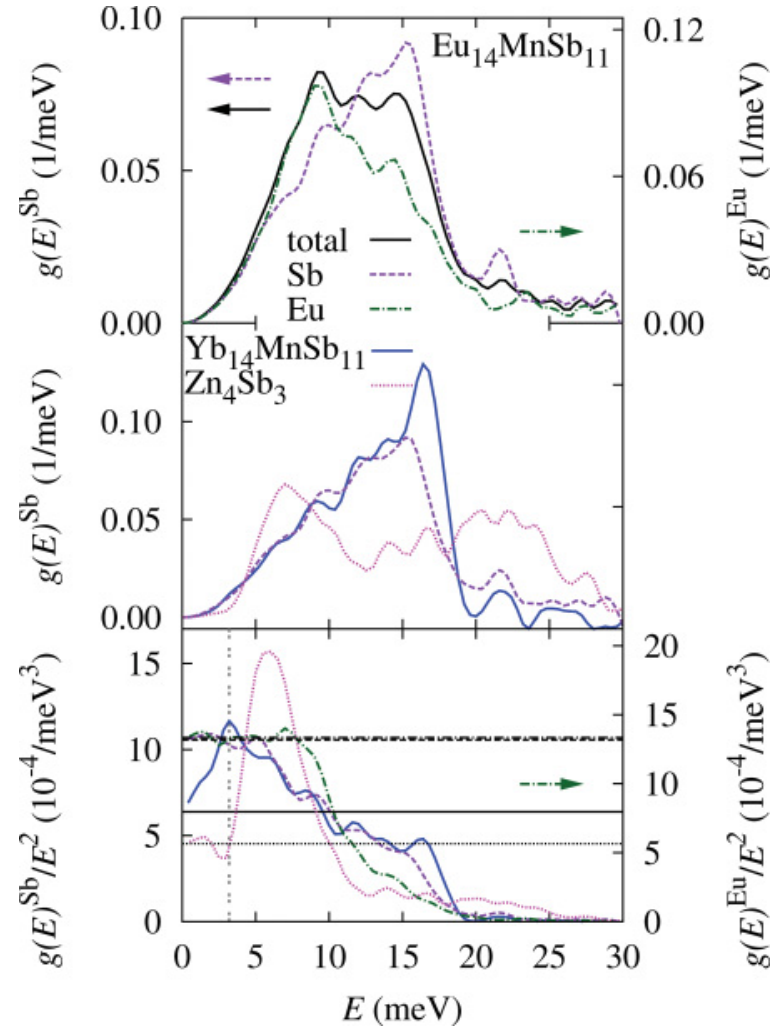

FIG. 9. (Color online) (top) The partial and total DPS in $\mathrm{Eu}_{14} \mathrm{MnSb}_{11}$ obtained from the $\mathrm{Sb}$ and Eu NIS spectra. The (left) $\mathrm{Sb}$ and (right) Eu scales are scaled to the $\mathrm{Sb}$ and Eu resonant masses; see text. (middle) The partial Sb DPS in $\mathrm{Yb}_{14} \mathrm{MnSb}_{11}, \mathrm{Eu}_{14} \mathrm{MnSb}_{11}$, and $\mathrm{Zn}_{4} \mathrm{Sb}_{3}$. (bottom) The reduced DPS and the Debye levels, indicated as horizontal lines in the same line style, obtained in the energy range between 0 and $4 \mathrm{meV}$ and for $\mathrm{Sb}$ in $\mathrm{Yb}_{14} \mathrm{MnSb}_{11}$ from the Debye temperature; see text. The gray dotted vertical line indicates the energy of the first Van Hove singularity as estimated from the transversal sound velocity; see text.

technique were used. The Eu phonon states in $\mathrm{Eu}_{14} \mathrm{MnSb}_{11}$ are in the same energy range as the ${ }^{121} \mathrm{Sb}$ states, but with a different distribution. A maximum at $8 \mathrm{meV}$ is observed in the Eu DPS and afterward the DPS decreases until $20 \mathrm{meV}$. The calculation of the velocity of sound is dependent on the mass of the resonant nucleus (see below), and therefore the DPS of $\mathrm{Sb}$ and $\mathrm{Eu}$ are scaled to the resonant nucleus mass in Fig. 9 in order to take the mass difference between $\mathrm{Sb}$ and $\mathrm{Eu}$ into account. The $\mathrm{Sb}$ and Eu DPS coincide in an energy range between 0 and $5 \mathrm{meV}$. Using the partial DPS, the total DPS was obtained by $g(E)=\left[11 g(E)^{\mathrm{Sb}}+14 g(E)^{\mathrm{Eu}}\right] / 25$ (see Fig. 9). The Mn contribution to the DPS was neglected. We assume that the Mn modes are mostly in the higher energy range, due to the small Mn mass. Because of the small number of $\mathrm{Mn}$ atoms in the unit cell, the influence on the lattice dynamics is minor. As observed in INS measurements the phonon modes are distributed over a very small energy region up to $19 \mathrm{meV}$ only, which indicates low force constants and a soft dynamic behavior. For comparison, the Sb DPS measured by NIS in the Zintl phase $\mathrm{Zn}_{4} \mathrm{Sb}_{3}$ is shown in Fig. 9. The $\mathrm{Sb}$ modes in $\mathrm{Zn}_{4} \mathrm{Sb}_{3}$ exhibit a very different shape and are in a different energy region compared to $\mathrm{Yb}_{14} \mathrm{MnSb}_{11}$. The peak observed 
at $\sim 7 \mathrm{meV}$ is consistent with the localized phonon mode observed in the neutron data. ${ }^{15}$

The mean force constant, $F_{m}$, can be calculated from the partial DPS with the mass $m_{R}$ of the resonant nucleus used for NIS measurements by ${ }^{40}$

$$
F_{m}=\frac{m_{R}}{\hbar} \int_{0}^{\infty} g(E) E^{2} d E,
$$

where $F_{m}$ is isotope specific and it is an average of all atom positions from the specific isotope. With this formula $F_{m}=$ $85(5) \mathrm{N} / \mathrm{m}$ for $\mathrm{Sb}$ in $\mathrm{Yb}_{14} \mathrm{MnSb}_{11}$ and $F_{m}=95(5) \mathrm{N} / \mathrm{m}$ and $F_{m}=107(5) \mathrm{N} / \mathrm{m}$ for Eu and $\mathrm{Sb}$ in $\mathrm{Eu}_{14} \mathrm{MnSb}_{11}$, respectively, were obtained. These values are small compared to other Zintl phases, e.g., $\mathrm{Zn}_{4} \mathrm{Sb}_{3}$, for which a $\mathrm{Sb}$ mean force constant of $131(2) \mathrm{N} / \mathrm{m}$ was obtained from the data. This indicates soft $\mathrm{Sb}$ and $\mathrm{Yb}$ bonds in $\mathrm{Yb}_{14} \mathrm{MnSb}_{11}$. From $g(E)$, the $\mathrm{Sb}$ Debye temperature, $\theta_{D}^{\mathrm{Sb}}$, for $\mathrm{Yb}_{14} \mathrm{MnSb}_{11}$ of $184(5) \mathrm{K}$ and the Eu Debye temperature, $\theta_{D}^{\mathrm{Eu}}$, in $\mathrm{Eu}_{14} \mathrm{MnSb}_{11}$ of $160(5) \mathrm{K}$ was obtained, in the high-temperature limit $T>\theta_{D}$, with the relation ${ }^{40}$

$$
\theta_{D}^{2}=\frac{3}{k_{B}^{2} \int_{0}^{\infty} g(E) d E / E^{2}} .
$$

The $\mathrm{Sb}$ Debye temperature in $\mathrm{Yb}_{14} \mathrm{MnSb}_{11}$ is in good agreement with $\theta_{D}^{\mathrm{Sb}}$ of 182(2) $\mathrm{K}$ obtained by Mössbauer spectroscopy measurements. ${ }^{29}$ The mean value of the Debye temperatures for $\mathrm{Sb}$ and Eu obtained by $\left(11 \theta_{D}^{\mathrm{Sb}}+14 \theta_{D}^{\mathrm{Eu}}\right) / 25$ is $170(5) \mathrm{K}$, in good agreement with the $\mathrm{Yb}_{14} \mathrm{MnSb}_{11} \theta_{D}$ of 160(10) K obtained by specific heat capacity measurements. ${ }^{19}$ This is also a proof that the $\mathrm{Yb}$ phonon modes are very similar to the Eu phonon modes and that the $\mathrm{Yb}$ Debye temperature has to be in the same magnitude as $\theta_{D}^{\mathrm{Eu}}$, because otherwise the macroscopic measured Debye temperature of $\mathrm{Yb}_{14} \mathrm{MnSb}_{11}$ would not be in agreement with the mean value of $\theta_{D}^{\mathrm{Sb}}$ and $\theta_{D}^{\mathrm{Eu}}$. It is expected that the $\mathrm{Mn}$ phonon modes are in an energy region between 20 and $25 \mathrm{meV}$ (see discussion below) and therefore the Mn phonon modes give only a small contribution to the Debye temperature, because $\theta_{D}$ is mainly dependent on the low-energy phonon modes [see Eq. (3)]. The large density alone does not explain the low Debye temperature and the low velocity of sound in $\mathrm{Yb}_{14} \mathrm{MnSb}_{11}$, because some materials with a similar density, such as $\mathrm{FeSb}_{2}, \rho=8.14 \mathrm{~g} /\left(\mathrm{cm}^{3}\right),{ }^{45}$ exhibit a much larger Debye temperature ${ }^{46}$ of $\sim 300 \mathrm{~K}$, and in contrast $\mathrm{Eu}_{14} \mathrm{MnSb}_{11}$ exhibits both a much lower density and a lower velocity of sound. The low Debye temperature is in accordance with the small phonon mode cutoff energy, as was also observed ${ }^{39}$ in $\mathrm{La}_{3} \mathrm{Te}_{4}$, another high-temperature thermoelectric material, with a phonon cutoff energy of $\sim 18 \mathrm{meV}$ and a Debye temperature of $173 \mathrm{~K}$. A Sb Debye temperature for $\mathrm{Zn}_{4} \mathrm{Sb}_{3}$ of $193(1) \mathrm{K}$ was obtained from $g(E)^{\mathrm{Sb}}$, which is in good agreement with the average Sb Mössbauer temperature $^{47}$ of $200(5) \mathrm{K}$. From the Debye level in the reduced DPS (see bottom panel of Fig. 9), the mean value of the sound velocity can be obtained ${ }^{38}$ by $\lim _{E \rightarrow 0}\left[g(E) / E^{2}\right]=$ $m_{R} /\left(2 \pi^{2} \rho \hbar^{3} v_{s}^{3}\right)$, with the densities, $\rho$, of $8.35(2) \mathrm{g} / \mathrm{cm}^{3}$ for $\mathrm{Yb}_{14} \mathrm{MnSb}_{11}$, of $6.87 \mathrm{~g} / \mathrm{cm}^{3}$ for $\mathrm{Eu}_{14} \mathrm{MnSb}_{11}$ from Ref. 48 , and of $6.36 \mathrm{~g} / \mathrm{cm}^{3}$ for $\mathrm{Zn}_{4} \mathrm{Sb}_{3}$ from Ref. 13. The sound velocity for $\mathrm{Eu}_{14} \mathrm{MnSb}_{11}$ obtained from the Eu DPS is $1690(50) \mathrm{m} / \mathrm{s}$, which is in good agreement with the value obtained from the $\mathrm{Sb}$ DPS. From the Sb DPS of $\mathrm{Yb}_{14} \mathrm{MnSb}_{11}$ a sound velocity of $1700(200) \mathrm{m} / \mathrm{s}$ was obtained. The large error arises from the uncertainty in the determination of the Debye level from the $\mathrm{Sb}$ DPS. In this case a more precise estimation of the sound velocity was obtained with the Debye approximation ${ }^{49}$ $v_{s} \approx\left(k_{B} \theta_{D}\right) /\left[\hbar\left(6 \pi^{2} N\right)^{1 / 3}\right]=1880(50) \mathrm{m} / \mathrm{s}$, and using the density of atoms $N=3.4 \times 10^{28}$ atoms $/ \mathrm{m}^{3}$ for $\mathrm{Yb}_{14} \mathrm{MnSb}_{11}$. The Debye level was calculated from this sound velocity and is indicated in Fig. 9. This sound velocity is in the same range as for $\mathrm{PbTe}$ and $\mathrm{Bi}_{2} \mathrm{Te}_{3}$, for which mean sound velocities of 1840 and $1770 \mathrm{~m} / \mathrm{s}$, respectively, were calculated from literature values, ${ }^{50,51}$ and small compared to $\mathrm{Zn}_{4} \mathrm{Sb}_{3}$ for which a sound velocity of $2300(100) \mathrm{m} / \mathrm{s}$ was obtained from the $\mathrm{Sb}$ DPS. The value for $\mathrm{Zn}_{4} \mathrm{Sb}_{3}$ is in good agreement with the value of $2470 \mathrm{~m} / \mathrm{s}$ obtained ${ }^{23}$ from the elastic constants. The differences in the DPS and the thermodynamic properties of $\mathrm{Yb}_{14} \mathrm{MnSb}_{11}$ and $\mathrm{Zn}_{4} \mathrm{Sb}_{3}$ indicate the different mechanisms leading to the low thermal conductivity observed in Zintl phases: In $\mathrm{Yb}_{14} \mathrm{MnSb}_{11}$ a low sound velocity, associated with soft interatomic bonds and the low effective heat capacity contribution due to the larger unit cell volume as compared to $\mathrm{Zn}_{4} \mathrm{Sb}_{3}$, are the reasons for the low thermal conductivity. In contrast, in the Sb DPS of $\mathrm{Zn}_{4} \mathrm{Sb}_{3}$ a strong localized phonon mode is observed and this mode can be related to the proposed mechanism of the reduction of the acoustic phonon mean free path by vibrational subunits, ${ }^{15}$ which are proposed to be a mechanism for the low thermal conductivity in $\mathrm{Zn}_{4} \mathrm{Sb}_{3}$, in addition to the $\mathrm{Zn}$ disorder and nanopores ${ }^{13,52}$ observed in $\mathrm{Zn}_{4} \mathrm{Sb}_{3}$, which were also related to a low phonon mean free path.

With the sound velocity of $1880(50) \mathrm{m} / \mathrm{s}$ for $\mathrm{Yb}_{14} \mathrm{MnSb}_{11}$ and the Poisson ratio ${ }^{32}$ of 0.24 , a longitudinal and transversal sound velocity was calculated to 2900(10) and $1700(10) \mathrm{m} / \mathrm{s}$, respectively. From the transversal sound velocity we estimated that the first Van Hove singularity appears in the DPS at $\sim 3 \mathrm{meV}$ (see dotted vertical line in Fig. 9). Applying the kinetic gas theory model, one obtains the lattice thermal conductivity $\kappa_{L}=1 / 3 C_{V} v_{s}^{2} \tau$, where $\tau$ is the phonon lifetime. In order to estimate the phonon lifetime in $\mathrm{Yb}_{14} \mathrm{MnSb}_{11}$ at ambient temperature, we used the macroscopically measured ${ }^{7}$ thermal conductivity at $300 \mathrm{~K}$ of $0.8 \mathrm{~W} /(\mathrm{m} \mathrm{K})$, the sound velocity of $1880(50) \mathrm{m} / \mathrm{s}$, and the specific heat capacity of $650 \mathrm{~J} \mathrm{~K}^{-1} \mathrm{~mol}^{-1}$ as obtained by the Dulong-Petit law. However, this estimate largely depends on whether the heat capacity of all phonons or only of the acoustic modes will contribute to $\kappa_{L}$. Within these limits the phonon lifetime is $0.16<\tau<50 \mathrm{ps}$, but clearly in this case it is not appropriate to infer the lifetime by these simple assumptions. Note that as the total lattice thermal conductivity is very low, the optical phonons may still contribute to a large fraction of this residual low thermal conduction. The specific heat capacity, $C_{V}$, can be calculated from the DPS ${ }^{40}$ in the harmonic approximation. The calculated $C_{V}$ is in good agreement with the lattice part of the experimental $C_{p}$ from the literature ${ }^{19,53}$ (see Fig. 10) and also in good agreement with the value of $650 \mathrm{~J} \mathrm{~K}^{-1} \mathrm{~mol}^{-1}$ obtained by the Dulong-Petit law. The peak at $50 \mathrm{~K}$ in the macroscopically measured $C_{p}$ is due to a magnetic transition. The difference between the macroscopic measurement or the value obtained by the Dulong-Petit law and the value obtained from NIS is due to the Mn contribution to $C_{V}$, which was not taken into account for the NIS value. The atomic 


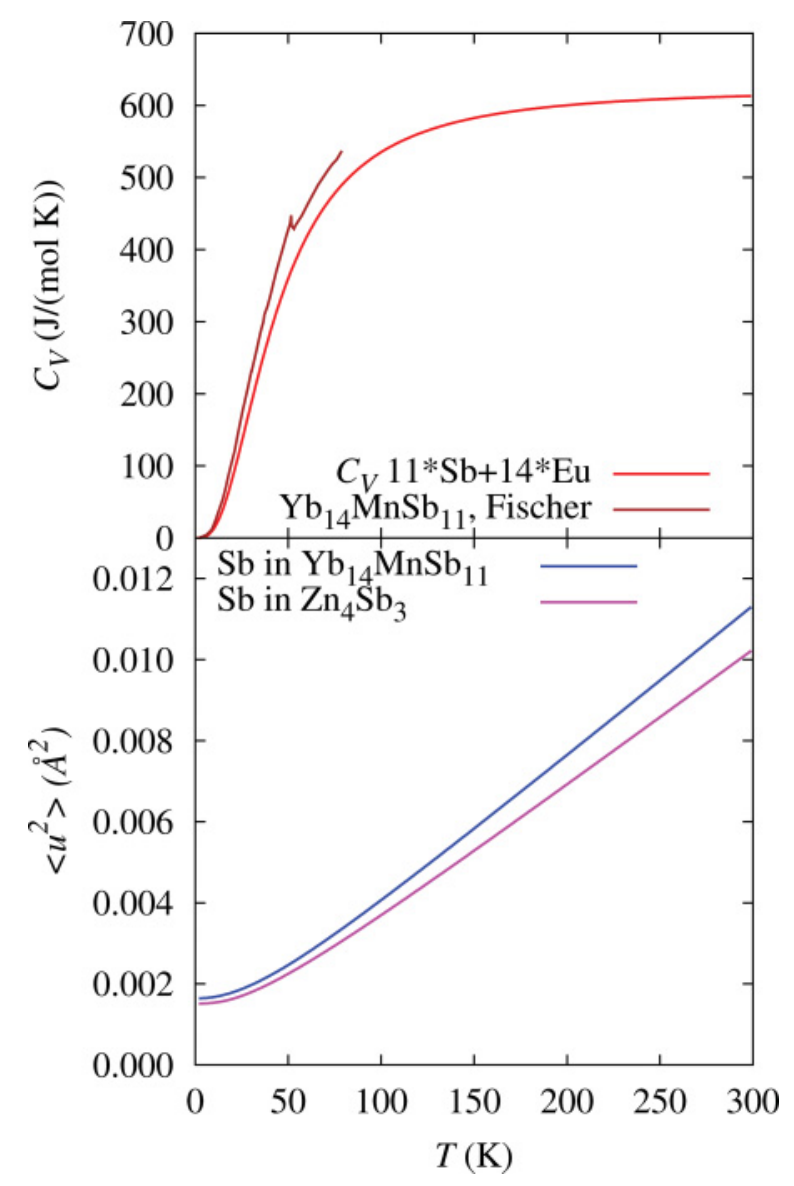

FIG. 10. (Color online) (top) The specific heat capacity obtained by NIS measurements (red) obtained by adding the heat capacities of $\mathrm{Sb}$ and $\mathrm{Eu}$, and macroscopic measurements of $\mathrm{Yb}_{14} \mathrm{MnSb}_{11}$ (brown) from Ref. 19. The peak in the macroscopic measurements at $50 \mathrm{~K}$ is due to a magnetic phase transition. (bottom) The mean square displacements for $\mathrm{Sb}$ in $\mathrm{Yb}_{14} \mathrm{MnSb}_{11}$ and $\mathrm{Zn}_{4} \mathrm{Sb}_{3}$ obtained by NIS.

mean square displacement, $\left\langle u^{2}\right\rangle$, can be obtained by $\left\langle u^{2}\right\rangle=$ $-\ln \left(f_{\mathrm{LM}}\right) / k^{2}$, where $k$ is the incident wave vector ${ }^{40}$ (see Fig. 10). The temperature dependence of $\left\langle u^{2}\right\rangle$ can be calculated from the Lamb-Mössbauer factor, $f_{\mathrm{LM}}(T)$, which is obtained in the harmonic approximation of the DPS by

$f_{\mathrm{LM}}(T)=\exp \left(-E_{R} \int_{0}^{\infty} \frac{g(E)}{E} \frac{1+\exp \left[-E /\left(k_{B} T\right)\right]}{1-\exp \left[-E /\left(k_{B} T\right)\right]} d E\right)$.

The slope of the $\mathrm{Sb}\left\langle u^{2}\right\rangle$ in $\mathrm{Yb}_{14} \mathrm{MnSb}_{11}$ is larger than in $\mathrm{Zn}_{4} \mathrm{Sb}_{3}$. As discussed above, the $\left\langle u^{2}\right\rangle$ in $\mathrm{Yb}_{14} \mathrm{MnSb}_{11}$ could not be obtained by our $\mathrm{x}$-ray diffraction pattern refinement. We suggest that precise $\left\langle u^{2}\right\rangle$ values can be obtained by pair distribution function measurements.

By combining the INS and NIS data, a complete picture of the density of states can be obtained. In Fig. 11 the comparison of the full DPS, measured by INS at FOCUS, with the neutron cross-section weighted partial DPS, obtained from NIS, is shown. Even by using the measurement on the Eu resonance for $\mathrm{Yb}$, a good agreement is observed. We expect that the Mn phonon modes are mostly at the higher-energy

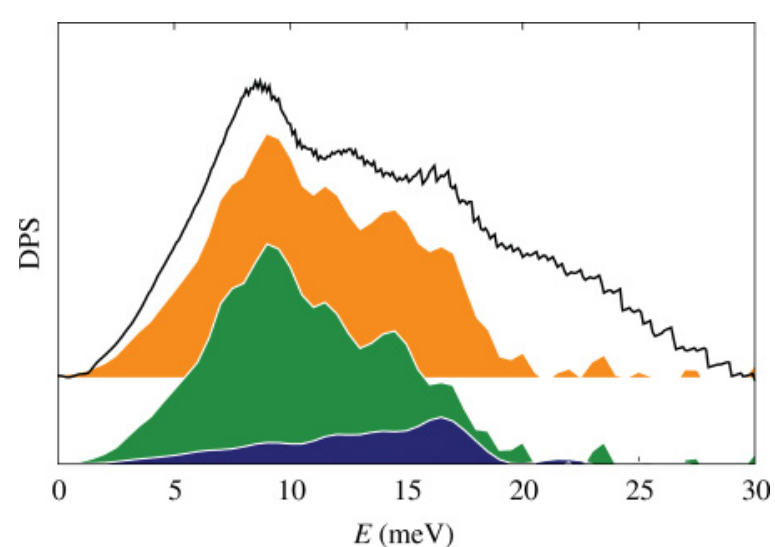

FIG. 11. (Color online) Comparison of the $\mathrm{Yb}_{14} \mathrm{MnSb}_{11}$ DPS extracted from INS (black line) and neutron cross-section weighted partial DPS obtained from NIS data, measured at the ${ }^{151}$ Eu resonance in $\mathrm{Eu}_{14} \mathrm{MnSb}_{11}$ (green) and at the ${ }^{121} \mathrm{Sb}$ resonance in $\mathrm{Yb}_{14} \mathrm{MnSb}_{11}$ (blue). Combining the green and blue area, the orange area is obtained.

end between 20 and $25 \mathrm{meV}$ due to the smaller Mn mass with respect to $\mathrm{Yb}$ and $\mathrm{Sb}$. One may compare this situation with the situation in the skutterudite $\mathrm{FeSb}_{3}$. In the partial DPS of $\mathrm{FeSb}_{3}$ it was observed ${ }^{36}$ that the major weight in the $\mathrm{Fe}$ DPS is located in strongly localized modes at larger energies than for Sb. Fe has a similar mass compared to $\mathrm{Mn}$, and in $\mathrm{FeSb}_{3} \mathrm{Fe}$ is also located in a $\mathrm{Sb}$ polyhedron, as $\mathrm{Mn}$ is in $\mathrm{Yb}_{14} \mathrm{MnSb}_{11}$, with similar Fe-Sb and $\mathrm{Mn}-\mathrm{Sb}$ distances ${ }^{17}$ of 2.6 and $2.75 \AA$, respectively. Similar force constants, and thus similar average vibrational energies, are expected. However, we cannot give direct experimental evidence by the techniques at hand, because $\mathrm{Mn}$ is not a suitable element for the NIS technique and the neutron cross section and the concentration of $\mathrm{Mn}$ atoms in the structure are small compared to $\mathrm{Yb}$ and $\mathrm{Sb}$, so that only $\sim 2 \%$ of the total inelastic neutron scattering signal is due to Mn. Furthermore, the FWHM of the resolution in the INS measurements is larger in the high-energy range compared to the low-energy range and the background subtraction is not precise, so the signal observed in the DPS above $20 \mathrm{meV}$ could be background.

\section{CONCLUSION}

The full partial DPS of $\mathrm{Yb}_{14} \mathrm{MnSb}_{11}$ is obtained by comparing NIS and INS data and reveals that the low-energy optical modes are mainly related to the dynamics of Eu in $\mathrm{Eu}_{14} \mathrm{MnSb}_{11}$ and of $\mathrm{Yb}$ in $\mathrm{Yb}_{14} \mathrm{MnSb}_{11}$ and that the $\mathrm{Sb}$ modes exhibit a linear energy dependence up to a cutoff energy of $20 \mathrm{meV}$. The acoustic phonon modes are below $5 \mathrm{meV}$. We have assumed that the Mn contribution to the DPS is negligible below $20 \mathrm{meV}$ and that $\mathrm{Mn}$ has an insignificant influence on the lattice dynamics. According to our INS and NIS data all phonon modes in $\mathrm{Yb}_{14} \mathrm{MnSb}_{11}$ are below $25 \mathrm{meV}$. The estimated mean force constant of $85 \mathrm{~N} / \mathrm{m}$ is related to the low energy of the phonon modes and indicates soft interatomic bonds. The sound velocity in $\mathrm{Yb}_{14} \mathrm{MnSb}_{11}$ of $v_{s}=1880(50) \mathrm{m} / \mathrm{s}$ is larger than in $\mathrm{Eu}_{14} \mathrm{MnSb}_{11}\left(v_{s}=1690(50) \mathrm{m} / \mathrm{s}\right)$; however, it is low compared to other antimonides like $\mathrm{Zn}_{4} \mathrm{Sb}_{3}$, for 
which a $v_{s}$ of $2300(100) \mathrm{m} / \mathrm{s}$ was obtained from the density of phonon states. In addition to the mechanism of the thermal conductivity reduction by a low effective heat capacity contribution, ${ }^{8}$ the low sound velocity is also a reason for the low thermal conductivity in $\mathrm{Yb}_{14} \mathrm{MnSb}_{11}$. Combined with the good electrical properties, this system is a candidate for thermoelectric applications. In the Sb density of phonon states of $\mathrm{Zn}_{4} \mathrm{Sb}_{3}$, a peak near $7 \mathrm{meV}$ is observed, which is consistent with the localized phonon mode observed in the neutron data ${ }^{15}$ and can be related to the proposed lowering of the phonon mean free path and the low thermal conductivity in $\mathrm{Zn}_{4} \mathrm{Sb}_{3}$. It thus appears that different mechanisms lead to the low thermal conductivities in $\mathrm{Yb}_{14} \mathrm{MnSb}_{11}$ and $\mathrm{Zn}_{4} \mathrm{Sb}_{3}$. No significant softening of the material at high temperatures up to $1200 \mathrm{~K}$ was observed in $\mathrm{Yb}_{14} \mathrm{MnSb}_{11}$, which is in line with the high melting temperature. This behavior has to be verified by other techniques, for example resonant ultrasound spectroscopy at high temperatures.

\section{ACKNOWLEDGMENTS}

We thank D. Bessas for his support during the NIS measurements, Dr. M. M. Koza for helpful discussions, and Professor Gary J. Long and Professor F. Grandjean for their assistance with preliminary work on these Zintl phases. The European Synchrotron Radiation Facility (ESRF), Grenoble, France; the Advanced Photon Source (APS), Argonne National Laboratory, Argonne, USA; the Swiss spallation neutron source SINQ, Paul Scherrer Institute, Villigen, Switzerland; and the Institut Laue-Langevin (ILL), Grenoble, France, are acknowledged for synchrotron and neutron beam time at the nuclear resonance station ID18 and ID22N, the 6ID-D high-energy station, the cold-neutron time-of-flight spectrometer FOCUS, and the IN6 spectrometer, respectively. R.H. acknowledges support from the Helmholtz-University Young Investigator Group "Lattices Dynamics in Emerging Functional Materials." *r.hermann@fz-juelich.de

${ }^{1}$ S. M. Kauzlarich, S. R. Brown, and G. J. Snyder, Dalton Trans. 21, 2099 (2007).

${ }^{2}$ F. J. DiSalvo, Science 285, 703 (1999).

${ }^{3}$ B. C. Sales, Science 295, 1248 (2001).

${ }^{4} \mathrm{G}$. A. Slack, CRC Handbook of Thermoelectrics (CRC Press, Boca Raton, FL, 1995), pp. 407-440.

${ }^{5}$ G. J. Snyder and E. S. Toberer, Nat. Mater. 7, 105 (2008).

${ }^{6}$ G. J. Snyder, Appl. Phys. Lett. 84, 2436 (2004).

${ }^{7}$ S. R. Brown, S. M. Kauzlarich, F. Gascoin, and G. J. Snyder, Chem. Mater. 18, 1873 (2006).

${ }^{8}$ E. S. Toberer, A. F. May, and G. J. Snyder, Chem. Mater. 22, 624 (2010).

${ }^{9}$ B. C. Sales, D. Mandrus, and R. K. Williams, Science 272, 1325 (1996).

${ }^{10}$ B. C. Sales, B. C. Chakoumakos, R. Jin, J. R. Thompson, and D. Mandrus, Phys. Rev. B 63, 245113 (2001).

${ }^{11}$ M. Christensen, A. B. Abrahamsen, N. B. Christensen, F. Juranyi, N. H. Andersen, K. Lefmann, J. Andreasson, C. R. H. Bahl, and B. B. Iversen, Nat. Mater. 7, 811 (2008).

${ }^{12}$ M. M. Koza, M. R. Johnson, R. Viennois, H. Mutka, L. Girard, and D. Ravot, Nat. Mater. 7, 805 (2008).

${ }^{13}$ G. J. Snyder, M. Christensen, E. Nishibori, T. Caillat, and B. B. Iversen, Nat. Mater. 3, 458 (2004).

${ }^{14}$ G. P. Meisner, D. T. Morelli, S. Hu, J. Yang, and C. Uher, Phys. Rev. Lett. 80, 3551 (1998).

${ }^{15}$ W. Schweika, R. P. Hermann, M. Prager, J. Persson, and V. Keppens, Phys. Rev. Lett. 99, 125501 (2007).

${ }^{16}$ N. R. Dilley, E. D. Bauer, M. B. Maple, S. Dordevic, D. N. Basov, F. Freibert, T. W. Darling, A. Migliori, B. C. Chakoumakos, and B. C. Sales, Phys. Rev. B 61, 4608 (2000).

${ }^{17}$ J. Y. Chan, M. M. Olmstead, S. M. Kauzlarich, and J. Webb, Chem. Mater. 10, 3583 (1998).

${ }^{18}$ G. A. Slack, Solid State Physics 34, edited by H. Ehrenreich (Academic, New York, 1979), pp. 1-71.

${ }^{19}$ I. R. Fisher, T. A. Wiener, S. L. Bud'ko, P. C. Canfield, J. Y. Chan, and S. M. Kauzlarich, Phys. Rev. B 59, 13829 (1999).
${ }^{20}$ E. Fabiani, A. Fontana, and U. Buchenau, J. Chem. Phys. 128, 244507 (2008).

${ }^{21}$ K. S. Burch, E. E. M. Chia, D. Talbayev, B. C. Sales, D. Mandrus, A. J. Taylor, and R. D. Averitt, Phys. Rev. Lett. 100, 026409 (2008).

${ }^{22}$ T. Caillat, J.-P. Fleurial, and A. Borshchevsky, J. Phys. Chem. Solids 58, 1119 (1997).

${ }^{23}$ S. Bhattacharya, R. P. Hermann, V. Keppens, T. M. Tritt, and G. J. Snyder, Phys. Rev. B 74, 134108 (2006).

${ }^{24}$ J. Nylén, M. Andersson, S. Lidin, and U. Häussermann, J. Am. Chem. Soc. 126, 16306 (2004).

${ }^{25}$ A. Le Bail, H. Duroy, and J. L. Fourquet, Mater. Res. Bull. 23, 447 (1988).

${ }^{26}$ J. Rodriguez-Carvajal, FULLPROF $V$ (2009) (Laboratoire Leon Brillouin (CEA-CNRS), France, 2009).

${ }^{27}$ R. T. Azuah, L. R. Kneller, Y. M. Qui, P. L. W. Tregenna-Piggott, C. M. Brown, J. R. D. Copley, and R. M. Dimeo, J. Res. Natl. Inst. Stand. Technol. 114, 341 (2009).

${ }^{28}$ D. Richard, M. Ferrand, and G. J. Kearley, J. Neutron Res. 4, 33 (1996) and [http://www.ill.eu/instruments-support/ computing-for-science/cs-software/all-software/lamp].

${ }^{29}$ R. P. Hermann, F. Grandjean, D. Kafle, D. E. Brown, C. E. Johnson, S. M. Kauzlarich, and G. J. Long, Inorg. Chem. 46, 10736 (2007).

${ }^{30}$ S. Kastbjerg, C. A. Uvarov, S. M. Kauzlarich, E. Nishibori, M. A. Spackman, and B. B. Iversen, Chem. Mater. 23, 3723 (2011).

${ }^{31}$ V. Ravi, S. Firdosy, T. Caillat, E. Brandon, K. Van der Walde, L. Maricic, and A. Sayir, J. Electron. Mater. 38, 1433 (2009).

${ }^{32}$ J. R. Gladden (private communication).

${ }^{33}$ T. Souma, G. Nakamoto, and M. Kurisu, J. Alloys Compd. 340, 275 (2002).

${ }^{34}$ J. K. D. Verma and M. D. Aggarwal, Solid State Commun. 8, 1929 (1970).

${ }^{35}$ D. T. Morelli, V. Jovovic, and J. P. Heremans, Phys. Rev. Lett. 101, 035901 (2008)

${ }^{36}$ A. Möchel, I. Sergueev, N. Nguyen, G. J. Long, F. Grandjean, D. C. Johnson, and R. P. Hermann, Phys. Rev. B 84, 064302 (2011).

${ }^{37}$ G. L. Squires, Introduction to the Theory of Thermal Neutron Scattering (Cambridge University Press, Cambridge, UK, 1978). 
${ }^{38}$ M. Y. Hu, W. Sturhahn, T. S. Toellner, P. D. Mannheim, D. E. Brown, J. Zhao, and E. E. Alp, Phys. Rev. B 67, 094304 (2003).

${ }^{39}$ O. Delaire, A. F. May, M. A. McGuire, W. D. Porter, M. S. Lucas, M. B. Stone, D. L. Abernathy, V. A. Ravi, S. A. Firdosy, and G. J. Snyder, Phys. Rev. B 80, 184302 (2009).

${ }^{40}$ R. Rüffer, and A. I. Chumakov, Hyperfine Interact. 128, 255 (2000).

${ }^{41}$ T. Matsunaga, N. Yamada, R. Kojima, S. Shamoto, M. Sato, H. Tanida, T. Uruga, S. Kohara, M. Takata, P. Zalden, G. Bruns, I. Sergueev, H.-C. Wille, R. P. Hermann, and M. Wuttig, Adv. Funct. Mater. 21, 2232 (2011).

${ }^{42}$ I. Sergueev, H.-C. Wille, R. P. Hermann, D. Bessas, Y. V. Shvyd'ko, M. Zajac, and R. Rüffer, J. Synchrotron Radiat. 18, 802 (2011).

${ }^{43}$ W. Sturhahn, T. S. Toellner, E. E. Alp, X. Zhang, M. Ando, Y. Yoda, S. Kikuta, M. Seto, C. W. Kimball, and B. Dabrowski, Phys. Rev. Lett. 74, 3832 (1995).

${ }^{44}$ V. G. Kohn and A. I. Chumakov, Hyperfine Interact. 125, 205 (2000).

${ }^{45}$ H. Holseth and A. Kjekshus, Acta Chem. Scand. 22, 3284 (1968).
${ }^{46}$ M. S. Diakhate, R. P. Hermann, A. Möchel, I. Sergueev, M. Søndergaard, M. Christensen, and M. J. Verstraete, Phys. Rev. B 84, 125210 (2011).

${ }^{47}$ R. P. Hermann, F. Grandjean, T. C. Chen, D. E. Brown, C. E. Johnson, G. J. Snyder, and G. J. Long, Inorg. Chem. 46, 767 (2007).

${ }^{48}$ A. Rehr and S. M. Kauzlarich, J. Alloys Compd. 207, 424 (1994).

${ }^{49}$ R. P. Hermann, F. Grandjean, and G. J. Long, Am. J. Phys. 73, 110 (2005).

${ }^{50}$ F. Ren, E. D. Case, J. R. Sootsman, M. G. Kanatzidis, H. J. Kong, C. Uher, E. Lara-Curzio, and R. M. Trejo, Acta Mater. 56, 5954 (2008).

${ }^{51}$ J. O. Jenkins, J. A. Rayne, and R. W. Ure, Phys. Rev. B 5, 3171 (1972).

${ }^{52}$ G. S. Pomrehn, E. S. Toberer, G. J. Snyder, and A. van de Walle, Phys. Rev. B 83, 094106 (2011).

${ }^{53}$ C. A. Cox, E. S. Toberer, A. A. Levchenko, S. R. Brown, G. J. Snyder, A. Navrotsky, and S. M. Kauzlarich, Chem. Mater. 23, 3723 (2011). 